\title{
Fingerprints of homogeneous nucleation and crystal growth in polyamide 66 as studied by combined infrared spectroscopy and fast scanning chip calorimetry
}

\author{
Arthur Markus Anton $^{1,2}$ (D) $\cdot$ Evgeny Zhuravlev $^{3,4}$ (D) $\cdot$ Wilhelm Kossack $^{1}$ (D) $\cdot$ Ruslan Andrianov $^{5} \cdot$ Christoph Schick $^{3,5}$ (D) \\ Friedrich Kremer ${ }^{1}$ iD
}

Received: 24 January 2020 / Revised: 23 April 2020 / Accepted: 23 April 2020 / Published online: 16 May 2020

(C) The Author(s) 2020

\begin{abstract}
Homogenous crystal nucleation and growth in polyamide 66 (PA66) are followed in situ by means of a combination of FTIR spectroscopy and fast scanning chip calorimetry (FSC). Therefore, a novel setup with a calorimetry chip equipped with an IRtransparent SiN membrane was developed, which enables to examine IR spectroscopic and FSC experiments on the identical specimen. Because of the small amount of sample material $(\sim 100 \mathrm{ng})$, it is possible to achieve heating and cooling rates up to $5000 \mathrm{Ks}^{-1}$, and hence to quench the sample into a fully amorphous state without quenched-in homogeneous crystal nuclei. Annealing the film then allows to determine the onset of homogenous nucleation and crystal growth by means of FSC, whereas molecular interactions are unraveled by FTIR spectroscopy. It is demonstrated that different moieties of PA66 respond distinctly during crystallization; far-reaching interactions such as hydrogen bonding are established prior to onset of short-range steric hindrance.
\end{abstract}

Keywords Spectroscopy · Optical · Infrared · Raman scattering · Calorimetry · Fast scanning calorimetry $\cdot$ Crystallization (polymer crystallization) $\cdot$ Homogeneous nucleation $\cdot$ Polyamides

Electronic supplementary material The online version of this article (https://doi.org/10.1007/s00396-020-04666-9) contains supplementary material, which is available to authorized users.

Arthur Markus Anton

arthur.anton@sheffield.ac.uk

1 Peter Debye Institute for Soft Matter Physics, Leipzig University, Linnéstr. 5, 04103 Leipzig, Germany

2 Department for Physics and Astronomy, The University of Sheffield, Hounsfield Road, Sheffield S3 7RH, UK

3 Institute of Physics and Competence Centre ${ }^{\circ} \mathrm{CALOR}$, University of Rostock, Albert-Einstein-Str. 23-24, 18059 Rostock, Germany

4 Department of Polymer Science and Engineering, School of Chemistry and Chemical Engineering, Shenzhen R\&D Center, State Key Laboratory of Coordination Chemistry, Nanjing University, Nanjing 210023, People's Republic of China

5 Institute of Chemistry, Kazan Federal University, Kazan 4200008, Russian Federation

\section{Introduction}

Polyamide 66 (PA66) is widely used as multipurpose thermoplastic polymer for fibers or applications in automotive and aeronautic [1]. Because PA66 is sensitive to the particular conditions during melt processing, which affects the resulting macroscopic properties and challenges precise manufacturing of high-end components [1], it is mandatory to pay attention to crystallization on the microscopic scale. It is generally believed that crystallization starts with an initial nucleation process within the supercooled sample followed by crystal growth $[2,3]$. Thereby, nucleation can be either homogeneous with nuclei solely formed by polymer chains through thermodynamic driving forces or heterogeneous with nuclei established at interfaces such as foreign materials or impurities. In general, polymer crystallization is based on heterogeneous nucleation. Studying homogenous nucleation in the bulk is only possible if heterogeneous nucleation is suppressed by sufficient fast cooling [2, 4, 5]. It is believed that supercooling (the difference between melting temperature and temperature of phase transformation) controls the number of 
nuclei and resulting crystal structure [6, 7]. At low supercooling (high transition temperature), a small number of nuclei and ordered triclinic $\alpha$-crystals are formed, which reversibly adopt a pseudo-hexagonal unit cell within the $\alpha^{\prime}$ form upon heating to the Brill transition temperature [8-10]. The creation of $\alpha$ - or $\alpha^{\prime}$-crystals at low supercooling is related to the formation of lamellae and spherulites [1]. At high supercooling, instead, a high number of crystal nuclei are formed and a less-ordered pseudo-hexagonal mesophase is established [1].

In order to study homogenous nucleation and crystal growth, fast heating and cooling is required. Thus, we employ non-adiabatic fast scanning calorimetry (FSC) which has been successfully established by Schick and coworkers as a method for analyzing polymer crystallization [11, 12]. As the cold crystallization enthalpy measured by means of FSC during heating or at isothermal conditions depends on the number of previously formed nuclei [13-15], FSC enables calorimetric analysis at heating and cooling rates up to $10^{6} \mathrm{Ks}^{-1}$ [16], much faster than in the case of standard differential scanning calorimetry (DSC) $[17,18]$ and to follow the development of crystals at a defined temperature deriving information concerning homogeneous nucleation and crystal growth [19].

In order to derive information about the structure and constraints of molecular moieties and their changes during the course of homogenous nucleation and crystal growth, Fourier-transform infrared (FTIR) spectroscopy is employed. Especially, the inherent specificity for vibrational modes of various molecular moieties and their sensitivity to conditions like local structure or environment emphasizes the importance of FTIR spectroscopy in following crystal nucleation and growth in situ [20-22]. This is realized based on a novel FSC-Chip having an IR-transparent $\mathrm{SiN}$ membrane, which enables in situ spectroscopic measurements by means of a fast scanning calorimeter modified to fit into the IR microscope of the spectrometer. On the basis of this novel setup, we are able

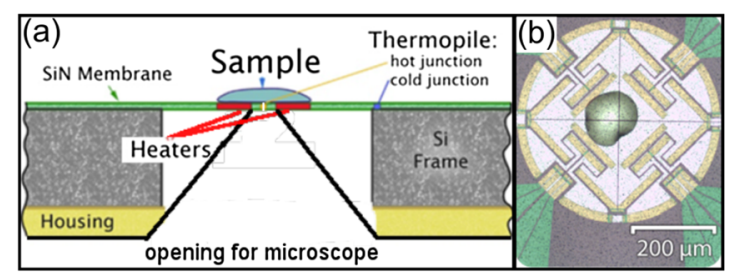

Fig. 1 a Geometry of the novel fast scanning calorimetry (FSC) chip with an IR-transparent membrane. $\mathbf{b}$ Micrograph of the chip with a sample on top. c Temperature trace for the combined IR and FSC measurements. First, PA66 is melt at $610 \mathrm{~K}$ (above the melting temperature $T_{\mathrm{m}}$, red dashed line) to erase thermal history before it is quenched with $5000 \mathrm{Ks}^{-1}$ to a defined annealing temperature $T_{\alpha}(318,323$, or $328 \mathrm{~K}$, to record FTIR spectra at the very same spot of the sample before, during, and after ultra-fast heat treatment. To the best of our knowledge, no study has been published about analyzing the FTIR signals of PA66 in situ during homogeneous nucleation and crystal growth, starting from a well-defined amorphous sample by means of FSC. The combination of FSC which allows exact control of nucleation and crystallization pathways with FTIR spectroscopy enabling analysis of molecular vibrations and structural changes in amorphous as well as crystalline phases allows us to study the crystallization of PA66 on a precisely prepared sample film.

\section{Materials and methods}

The sample material (polyamide 66, PA66) was purchased from DuPont de Nemours, Inc., (Zytel ${ }^{\circledR} 101 \mathrm{~L}, M_{\mathrm{n}}=$ 17,000-18,000 $\mathrm{gmol}^{-1}$ ) and used as supplied. In order to cool the sample at $5000 \mathrm{Ks}^{1}$, the specimen should be thinner than $10 \mu \mathrm{m}$ and weighing a few tens of nanograms. In addition, the thickness and area of the specimen must be appropriate to provide adequate absorbance in the IR spectral range. Thus, the sample was prepared directly on the calorimetric sensor (Fig. 1a, b) by melting PA66 using the internal sensor heater [23]. Molten PA66 was spread over the heater by a thin copper wire until the optimal thickness and film area for fast scanning calorimetry (FSC) and IR measurements were obtained. The sample preparation was performed under a microscope in a dry nitrogen atmosphere to avoid degradation of PA66.

For fast scanning calorimetry (FSC) experiments, a novel designed chip with an implemented IR-transparent membrane (100 $\mu \mathrm{m}$ diameter, Fig. 1a) was employed and a fast scanning calorimeter from the University of Rostock was modified in order to adjust it to the IR microscope of the spectrometer [23, 24]. After the sample was melted at $600 \mathrm{~K}$ for $0.1 \mathrm{~s}$, it was quenched to a defined temperature $(318,323$, or $328 \mathrm{~K})$ and

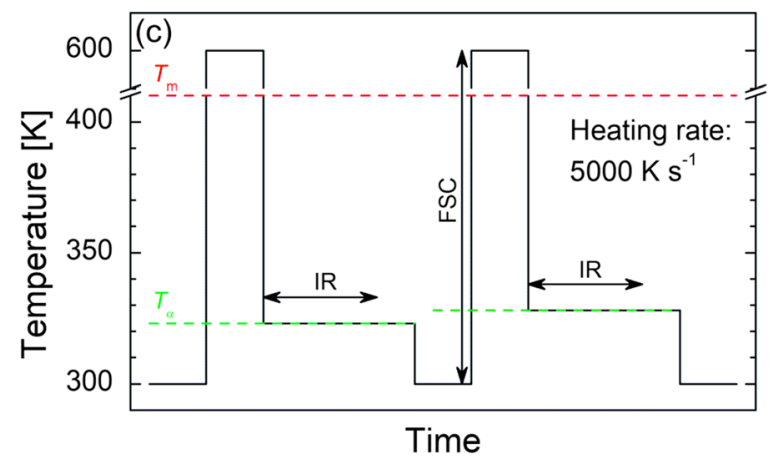

green dashed line). For IR measurements, the film is kept at $T_{\alpha}$ and IR spectra are recorded consecutively. Afterward, for FSC measurements, the film is quenched to $300 \mathrm{~K}$ after a defined crystallization time $\tau_{\alpha}$ and calorimetric measurements are examined during heating from 300 to $610 \mathrm{~K}$. After one annealing run at a particular temperature $T_{\alpha}$, the same scheme is examined with another $T_{\alpha}$ 
allowed to anneal isothermally for a particular time $\tau_{\alpha}$ at a defined temperature $T_{\alpha}$. Depending on the time at the isotherm, we obtain three major states of glass: fully amorphous glass, glass with homogeneous nuclei, and glass with crystals, as well as intermediate states [2]. After annealing, the specimen is quenched to $300 \mathrm{~K}$ and reheated at $5000 \mathrm{Ks}^{-1}$ for recording the enthalpy change. During all measurements, the chip was constantly flushed with dry nitrogen gas. The glass transition of amorphous PA66 is expected at around $333 \mathrm{~K}$ at $5000 \mathrm{Ks}^{1}$ [1, 25-27]. In this work, heating was performed from room temperature; therefore, we cannot observe the relatively broad glass transition step fully.

IR experiments were examined by means of a BioRad FTS 6000 FTIR spectrometer equipped with a UMA500 IR microscope and a mercury-cadmium-telluride (MCT) detector (Kolmar Technologies, Inc., USA). Spectra were continuously recorded with a spectral resolution of $1 \mathrm{~cm}^{-1}$ and a temporal resolution of $9.6 \mathrm{~s}$ immediately after the melted sample was quenched to the particular annealing temperature $(318,323$, or $328 \mathrm{~K}$ ). In order to record spectra from a sample with a higher degree of crystallinity (approx. 40\%), the film was in addition annealed at $470 \mathrm{~K}$ for a particular time and subsequently quenched to $300 \mathrm{~K}$ to freeze crystal growth [11, 28-30]. Afterward, FTIR spectra were recorded.

Concerning data evaluation, details regarding the integration procedure of the cold crystallization and melting peaks in the FSC curves are described elsewhere [19, 23]. For IR spectroscopy, the spectral region between 3520 and $2925 \mathrm{~cm}^{-1}$ has been modeled as a superposition of 6 Gaussians and a constant horizontal baseline. For the amide I and II bands, 3 asymmetric pseudo-Voigt profiles [31] have been fitted to the spectral region between 1800 and $1480 \mathrm{~cm}^{-1}$ after a polynomial of degree 2 was subtracted as the baseline. In the case of the scissoring vibrations, spectral features are less pronounced for amorphous samples and relative peak heights alter in the course of crystallization. Thus, in order to determine the peak positions, a horizontal baseline was subtracted between 1555 and $1370 \mathrm{~cm}^{-1}$ and a sum of 9 peaks ( 8 Gaussians and 1 pseudo-Voigt function for the peak at $\left.1474 \mathrm{~cm}^{-1}\right)$ was fitted to spectra of semi-crystalline samples ( $\sim 40 \%$ crystallinity). During the fitting of amorphous spectra, these positions were kept fixed. Please note, these particular peaks broaden during the Brill transition but maintain their spectral positions, as discussed by Cooper et al. [30]. Thus, the spectrum from the sample crystallized at $470 \mathrm{~K}$ (low supercooling, $\alpha$ mesophase) can be employed to determine the positions of the peaks arising from $\mathrm{CH}_{2}$ scissoring mode in the $\alpha$ ' mesophase (high supercooling).

In general, it is believed that crystal growth obeys the Kolmogorov-Johnson-Mehl-Avrami formalism [6]. In order to extract reliable information, the full course of crystallization is necessary [7], which was not accessible for this set of experiments. However, an empirical exponential growth appeared to provide the best fit to the recent data, potentially due to an Avrami exponent close to 1 as described by Bruna et al. [7] or Brucato et al. [32].

\section{Results and discussion}

Crystal nucleation and growth is determined on the basis of FSC. The heating curves starting from $300 \mathrm{~K}$ consist of the high-temperature part of the glass transition step, a cold crystallization and a melting peak (Fig. 2a) [4, 19, 33-35]. The interplay between these three contributions depends on the condition of the sample prior to reheating, namely quenching,
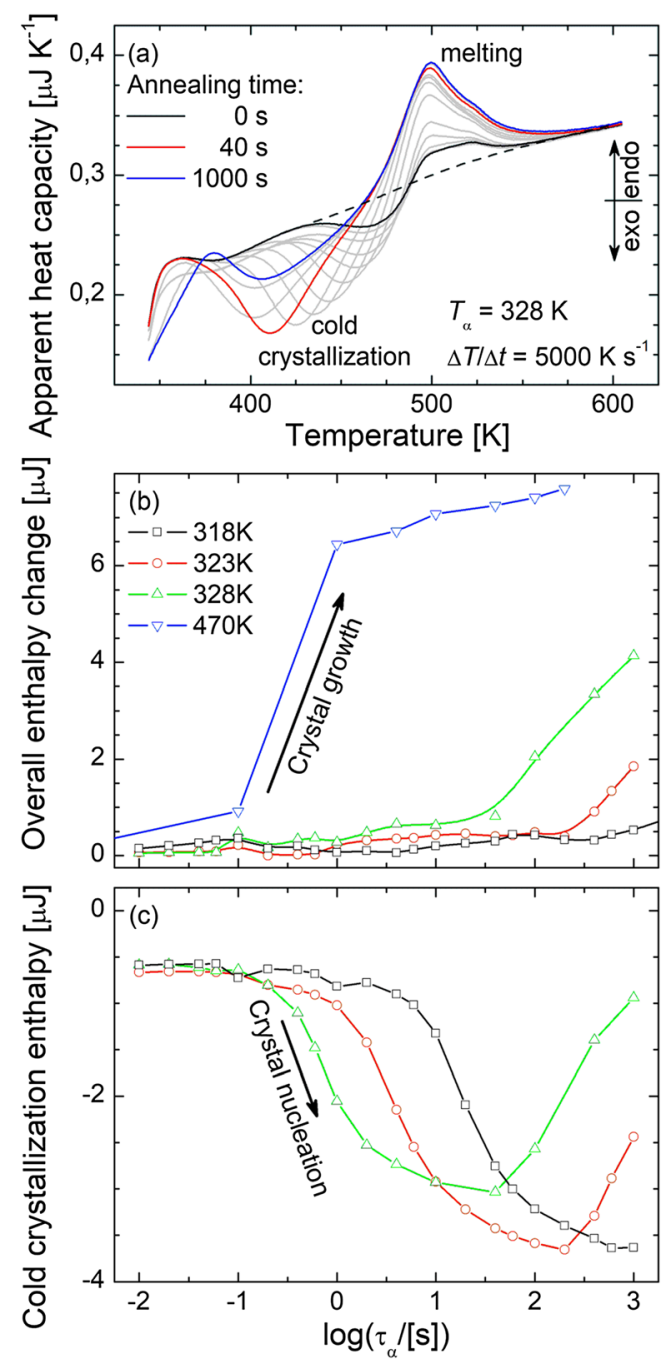

Fig. 2 a Fast scanning calorimetry signal and $\mathbf{b}$ determined overall enthalpy change as well as c cold crystallization enthalpy. The dashed line in a represents the baseline for peak integration (exemplary case of $0 \mathrm{~s}$ annealing time). The overall enthalpy change is determined as the sum of the positive melting and the negative cold crystallization enthalpies. Crystallization sets in at the deviation of the overall enthalpy change from 0 in $\mathbf{b}$ or the minimum of the cold crystallization enthalpy in $\mathbf{c}$. The blue curve in b represents the overall enthalpy change during full crystallization at high temperature 
isothermal nucleation, and crystal growth. In order to avoid reordering which can result in an overlap of exo- and endothermal processes fast heating is, therefore, mandatory $[2,19]$. Details regarding the analysis of the heating curve after annealing are provided in Ref. 2 and 42. The area under the curve (after baseline correction) of the positive melting peak, the negative cold crystallization peak, and the sum of both are denoted as melting enthalpy, cold crystallization enthalpy, and overall enthalpy change, respectively.

The overall enthalpy change (Fig. 2b) is related to crystallization during isothermal annealing. The increase in the overall enthalpy change represents crystal formation during annealing and melting during reheating. It is believed that the decrease of the cold crystallization enthalpy (Fig. 2c) prior to the increase of the overall enthalpy change indicates (homogeneous) crystal nucleation within this period of time $[11,28,29]$. After a particular annealing time, nucleation starts to be superimposed with crystal growth resulting in an increase of the cold crystallization enthalpy after passing a minimum. We experimentally determined that the optimal temperature range for homogeneous nucleation of PA66 is in between 318 and $328 \mathrm{~K}$. The temperature and time of homogeneous nuclei formation and growth are used as a guide to interpret the FTIR data. In particular for $T_{\alpha}=323 \mathrm{~K}$, the minimum of the cold crystallization enthalpy and the onset of crystal growth is at $\tau_{\alpha}=130 \mathrm{~s}\left(\log \left(\tau_{\alpha} /[s]\right)=2.1\right)$.

Within the IR spectra, peaks specific for structure-defining moieties can be identified. At around $3300 \mathrm{~cm}^{-1}$, the N-H stretching vibration $v(\mathrm{~N}-\mathrm{H})$ specific for the proton being incorporated into a hydrogen bond gives rise to a distinct band (Fig. 3b) [36, 37]. At lower frequencies, one can find the prominent amide I and II bands at around 1640 and $1550 \mathrm{~cm}^{-1}$ (Fig. $3 \mathrm{~d}$ ) assigned to $\mathrm{C}=\mathrm{O}$ stretching $v(\mathrm{C}=\mathrm{O})$ and $\mathrm{N}-\mathrm{H}$ bending vibrations $\delta(\mathrm{N}-\mathrm{H})$, respectively $[36,37]$.

Following their temporal evolution, FSC data predict that at $318 \mathrm{~K}$ crystal nucleation sets in at around $\log _{10}\left(\tau_{\alpha}\right)=0$ but crystal growth is below the level detectable by FSC during the first $1000 \mathrm{~s}$ (Fig. 2c). In the IR spectra, however, no significant alteration can be observed (Fig. 4). At $328 \mathrm{~K}$, instead, crystal growth sets in already during recording the very first spectrum. Thus, we analyze the crystal nucleation and growth at $323 \mathrm{~K}$ in more detail. Therefore, we divide the temporal trace into two parts: the crystal nucleation marked by the cold crystallization enthalpy at shorter times prior its maximum along with a zero overall enthalpy change and the crystal growth marked by a positive overall enthalpy change $\left(\tau_{\alpha}=130 \mathrm{~s}\right.$, dashed vertical line in Fig. 5).
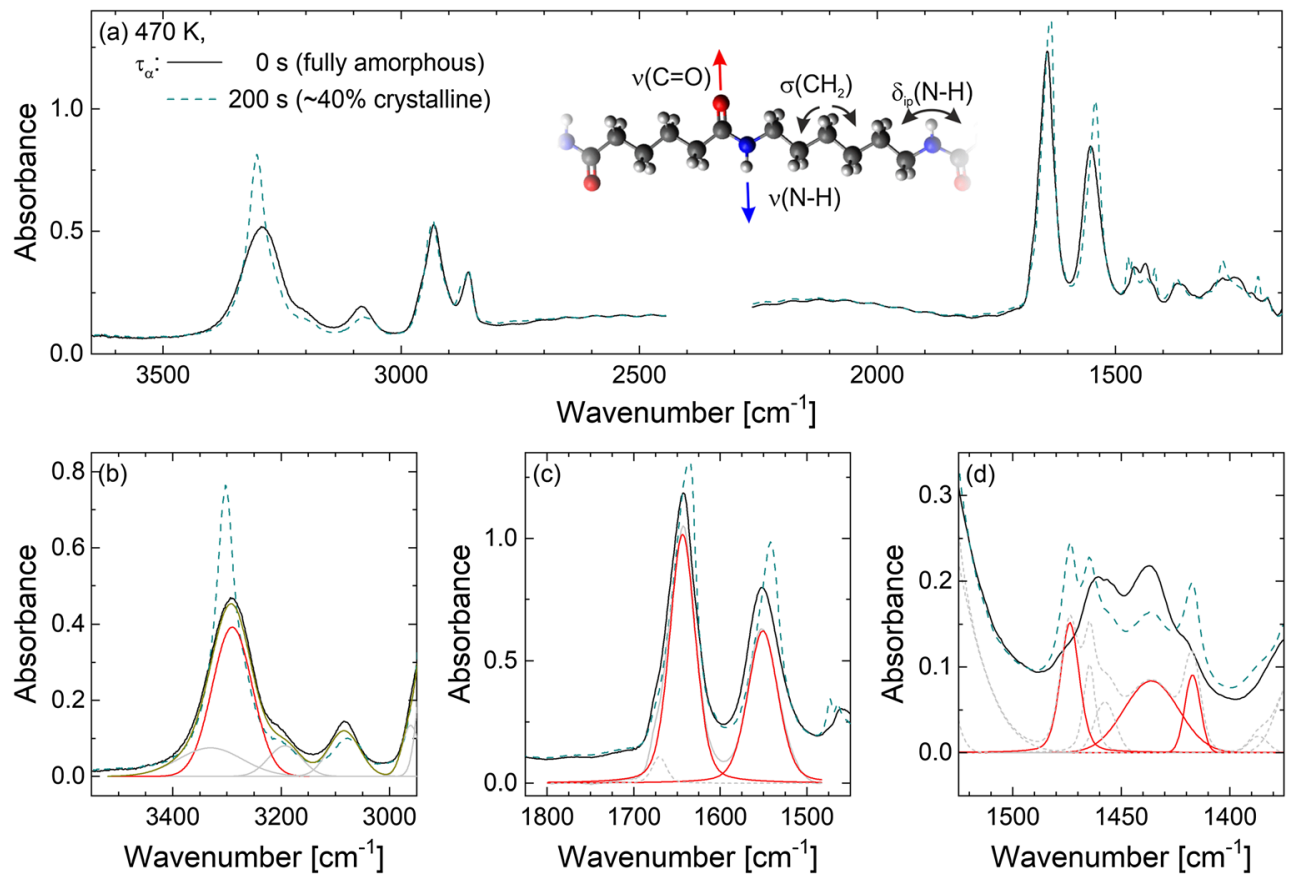

Fig. 3 a IR spectrum of the sample on the chip annealed at $470 \mathrm{~K}$ for $0 \mathrm{~s}$ resulting in a fully amorphous sample and $200 \mathrm{~s}$ resulting in approx. $40 \%$ crystallinity. After annealing, spectra are measured at $300 \mathrm{~K}$. b The spectral region of the $\mathrm{N}-\mathrm{H}$ stretching vibration $v(\mathrm{~N}-\mathrm{H})$ around $3300 \mathrm{~cm}^{-1}$ specific for protons incorporated into hydrogen bonds, $\mathbf{c}$ the amide $\mathrm{I}\left(1640 \mathrm{~cm}^{-1}\right)$ and amide II $\left(1550 \mathrm{~cm}^{-1}\right)$ region arising chiefly from $\mathrm{C}=\mathrm{O}$ stretching $\vee(\mathrm{C}=\mathrm{O}$ ) and in-plane $\mathrm{C}-\mathrm{N}$ bending $\delta(\mathrm{NH})$ (with a contribution of C-N stretching $v(\mathrm{C}-\mathrm{N}))$, respectively, as well as $\mathbf{d ~} \mathrm{CH}_{2}$ scissoring modes $\sigma\left(\mathrm{CH}_{2}\right)\left(1500-1400 \mathrm{~cm}^{-1}\right)$. Example oscillatory fits to $\mathbf{b}, \mathbf{c}$ the

amorphous or $\mathbf{d}$ semi-crystalline spectra are indicated. Solid lines denote peaks used for structural study, dashed lines peaks used in order to model the spectra. Please note, the spectra of the sample crystallized at $470 \mathrm{~K}$ are for assignment and visualization purposes. This sample $(470 \mathrm{~K}$; low supercooling) represents the $\alpha$ mesophase, whereas the other spectra (318, 323, and $328 \mathrm{~K}$; high supercooling) analyzed hereafter most probably represent the $\alpha$ ' mesophase. Spectra of the latter samples are recorded consecutively at the particular annealing temperature 
Fig. 4 a The frequency position and $\mathbf{b}$ the change of the integrated absorbance of the N-H stretching vibration $v(\mathrm{~N}-\mathrm{H})$ specific for protons incorporated into hydrogen bonds at different crystallization temperatures $T_{\alpha}$ along with $\mathbf{c}$ the frequency position and $\mathbf{d}$ change of the integrated absorbance of the $\mathrm{C}=\mathrm{O}$ stretching vibration $v(\mathrm{C}=\mathrm{O})$ of the amide $\mathrm{I}$ band. Especially these two parts of the amide group form intermolecular hydrogen bonds. While at $318 \mathrm{~K}$ the crystallization is widely suppressed, at $323 \mathrm{~K}$ its temporal trace can be followed and at $328 \mathrm{~K}$ it is saturated
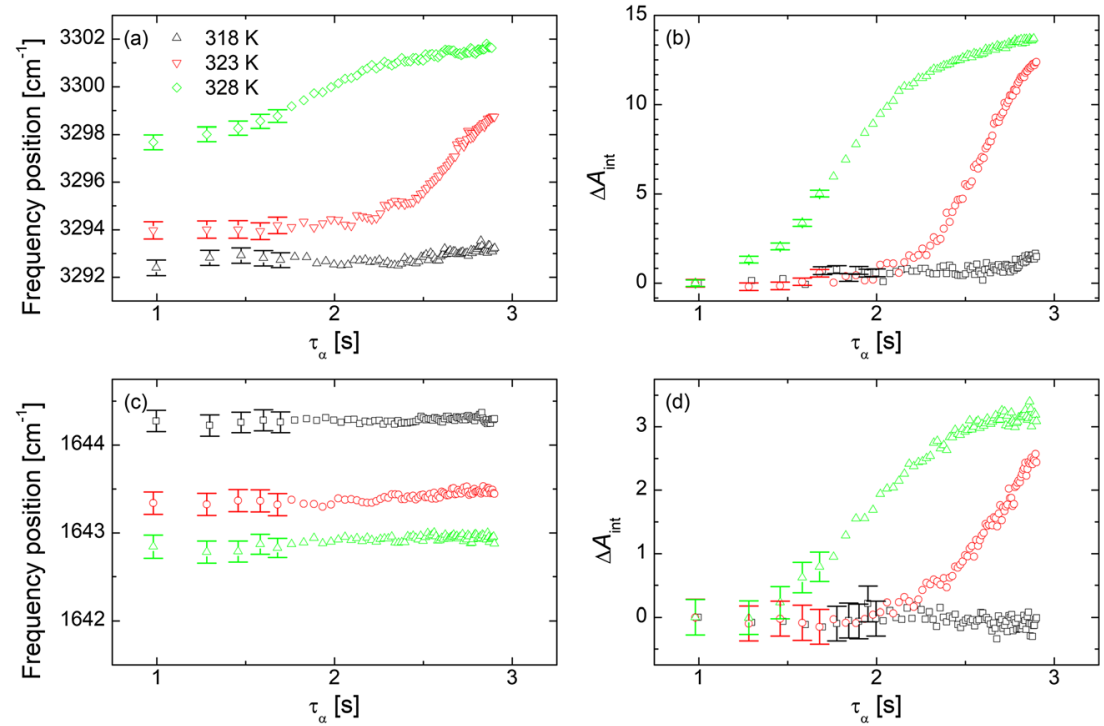

In the case of $v(\mathrm{~N}-\mathrm{H})$, it is evident that the vibration frequency is rapidly blue-shifted with the onset of crystal growth (Fig. 5c). Moreover, a slight increase is present already prior to crystal growth, namely, during crystal nucleation. This reflects a change in the potential of this vibrational mode already before crystallites are growing. In addition, the evolution of the frequency position exhibits a kink at the transition from homogeneous crystal nucleation to crystal growth. In the case of the integrated absorbance $A_{\text {int }}$, which is proportional to the number density of $\mathrm{H}$-bonds, it is slightly increasing during crystal nucleation. The trace bends off at the onset of crystal growth and evolves faster afterward (Fig. 5d).

In the case of $v(\mathrm{C}=\mathrm{O})$, the vibration frequency and the integrated absorbance (Fig. 5e, f) appear to be constant during crystal nucleation. It is widely believed that the secondary amine and the carbonyl part of different amide groups establish intermolecular hydrogen bonds [38]. Thus, a perturbation of the initial vibrational potential will affect the weaker $\mathrm{N}-\mathrm{H}$ single bond to a greater extent than the more stable $\mathrm{C}=\mathrm{O}$ double bond. However, when crystal growth sets in, the frequency and the integrated absorbance of $v(\mathrm{C}=\mathrm{O})$ are redshifted and decreased (Fig. 5e, f), respectively. For the sake of comparison, we hold the rate constants of $v(\mathrm{~N}-\mathrm{H})$ (frequency shift, 3.9975 $10^{-7} s^{-1}$; integrated absorbance, $1.46 \times 10^{-4} s^{-1}$, Table S1 in the Supporting Information) fixed and fit an exponential growth function to the data of $v(\mathrm{C}=\mathrm{O})$. On the one hand, the change of the vibration frequency can be hardly described with this rate constant (Fig.5e, Fig. S1 in the Supporting Information), even though an unrestricted fit results in a rate of $-4.2855 \times 10^{-6} s^{-1}$. On the other hand, the integrated absorbance does not obey the same rate (Fig. 5f; unrestricted fit, rate of $3.25 \times 10^{-6} s^{-1}$ ). Skrovanek and coworkers published that the frequency position of $v(\mathrm{~N}-\mathrm{H})$ is sensitive to the strength of hydrogen bonding but insensitive to conformation [39]. On the other hand, $v(\mathrm{C}=\mathrm{O})$ is a localized mode and sensitive to conformation through dipole-dipole interactions [39].

In general, annealing of glass gives rise to enthalpy relaxation, which might involve conformational changes [40]. In order to face the question, whether enthalpy relaxation is responsible for the changes in the IR spectra prior to the onset of crystal growth, we make use of the specificity of the IR absorption and distinguish and compare between stretching vibrations of the $\mathrm{N}-\mathrm{H}$ and the $\mathrm{C}=\mathrm{O}$ parts of the amide group. The former vibration is sensitive to the H-bond strength (comparable to the distance between binding partners), whereas it is insensitive to conformation (orientation of binding partners) [39]. In the case of the second vibration, this mode is sensitive to conformation due to dipole-dipole interaction but insensitive to the H-bond strength [39]. With regard to the experimental findings, the N-H stretching mode does exhibit a change during the annealing time, whereas the $\mathrm{C}=\mathrm{O}$ mode does not. Given the case of a potential conformational or structural change, it is highly unlikely that the amide groups as binding partners change their mutual distance (affecting the $\mathrm{N}-\mathrm{H}$ mode), but do not rotate (affecting the $\mathrm{C}=\mathrm{O}$ mode). In the general case, a rotation is less space- and energy-demanding in order to lower the overall energy of a system than a translation, especially when the amide parts are interconnected through highly flexible methylene groups. Thus, the experimental results contradict a conformational change during enthalpy relaxation.

In addition to the stretching vibrations $v(\mathrm{~N}-\mathrm{H})$ and $v(\mathrm{C}=\mathrm{O})$, we analyze the $\mathrm{N}-\mathrm{H}$ bending $\delta(\mathrm{N}-\mathrm{H})$ (with a contribution of $\mathrm{C}-\mathrm{N}$ stretching $v(\mathrm{C}-\mathrm{N})$ ) at the amide moiety giving rise to the amide II band. The integrated absorbance is only marginally affected during crystal nucleation and crystal growth as compared with the other vibrations (Fig.5h). This reflects that even during crystallization, the number density of the vibrating moieties is nearly constant; the rate constant of 


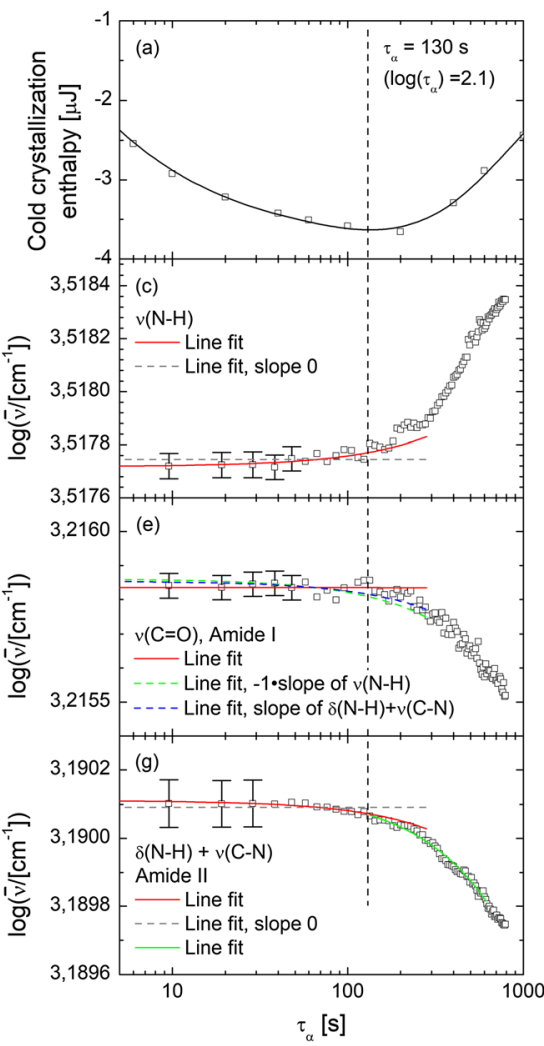

Fig. 5 a Cold crystallization enthalpy and $\mathbf{b}$ overall enthalpy change for crystallization at $323 \mathrm{~K}$ as well as $\mathbf{c}, \mathbf{e}, \mathbf{g}$, spectral position and $\mathbf{d}, \mathbf{f}, \mathbf{h}$ integrated absorbance of the $\mathbf{c}, \mathbf{d} \mathrm{NH}$ stretching vibration $v(\mathrm{~N}-\mathrm{H})$ specific for protons incorporated into hydrogen bonds, e, f $\mathrm{C}=\mathrm{O}$ stretching vibration, and $\mathbf{g}, \mathbf{h}$ the combination of $\delta(\mathrm{N}-\mathrm{H})+v(\mathrm{C}-\mathrm{N})$. The onset of crystal growth is determined as minimum of the cold crystallization enthalpy and marks the onset of the overall enthalpy change (dashed vertical line). Already prior the onset of the crystal growth, $v(\mathrm{~N}-\mathrm{H})$ and $\delta(\mathrm{N}-\mathrm{H})+v(\mathrm{C}-\mathrm{N})$ show changes of their vibration potential visible in a frequency shift. In the case of $v(\mathrm{C}=\mathrm{O})$, line fits at the vibrational frequency and integrated absorbance results in constants. The rates of

$v(\mathrm{~N}-\mathrm{H})$ does not represent the evolution of the $A_{\text {int }}$. A reason for this is that bending vibrations are more localized than stretching vibrations. On the other hand, the frequency position reflecting the vibration potential exhibits a kink already at $\tau_{\alpha} \approx 60 \mathrm{~s}$ (Fig. $5 \mathrm{~g}$, Fig. S2 in the Supporting Information). In contrast, the temporal evolution of $A_{\text {int }}$ remains irrespective of passing the onset of crystal growth (Fig. S2).

In contrast to the former vibrations located at the stiff and planar amide groups, the methylene scissoring modes $\sigma\left(\mathrm{CH}_{2}\right)$ arise from the butylene and hexylene parts interconnecting the amide moieties. Cooper et al. published that the specific position of each peak characterizes the location and the conformation of the particular vibrating $\mathrm{CH}_{2}$ groups [36]. On the basis of this, we identify peaks from $\mathrm{CH}_{2}$ groups in trans conformation adjacent to the $\mathrm{NH}$ and $\mathrm{C}=\mathrm{O}$ moiety of the amide group at 1474 and $1417 \mathrm{~cm}^{1}$ (Fig. 3d). In the case these methylene groups do not adopt a trans conformation, a band is supposed to appear at around $1440 \mathrm{~cm}^{-1}$, which matches well with a broad peak at $1436 \mathrm{~cm}^{1}$ from our study (Fig. 3c) [36].

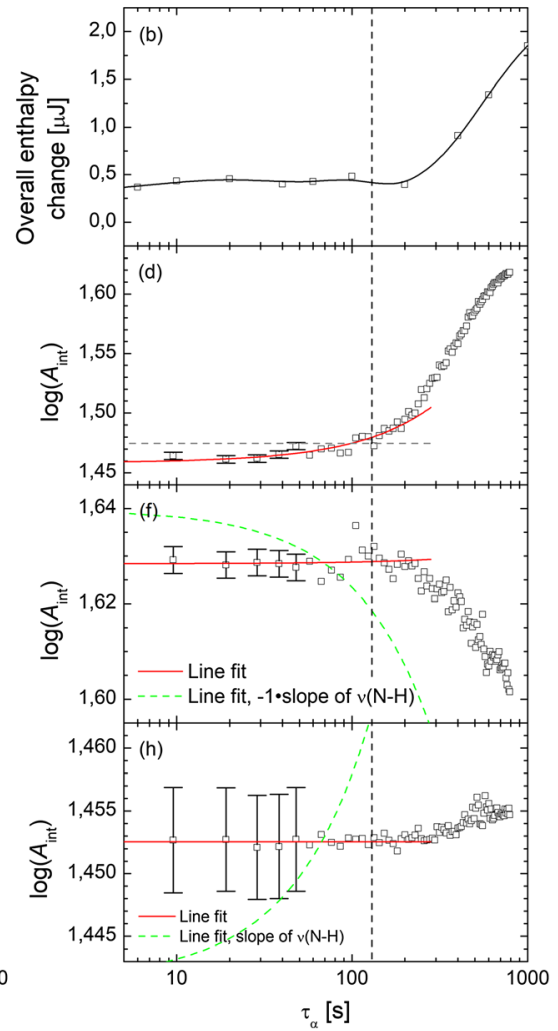

the frequency shift of $v(\mathrm{~N}-\mathrm{H})$ (panel c) or $\delta(\mathrm{NH})+v(\mathrm{C}-\mathrm{N})$ (panel g) fairly describe the rate of frequency shift of $v(\mathrm{C}=\mathrm{O})$ (panel e). The rate of the change of $v(\mathrm{~N}-\mathrm{H})$ (panel d), instead, does not describe the rates of $\nu(\mathrm{C}=\mathrm{O})$ (panel f) or $\delta(\mathrm{N}-\mathrm{H})+v(\mathrm{C}-\mathrm{N})$ (panel h). Constants (dashed horizontal lines) are guides for the eyes. Every line fit is based on the data either prior or after the onset of crystal growth. Please note the indicated error bars represent basically numerically uncertainties. Especially in panel $\mathbf{g}$, the scattering of points is much less than the numerical uncertainty. Thus, it can be treated as systematic uncertainty not affecting the qualitative assessment of results

Following the temporal evolution of the two peaks assigned to $\sigma\left(\mathrm{CH}_{2}\right)$ in trans conformation after the onset of crystal growth, the number density of the trans conformers increases while that of the non-trans conformers decreases, as it is to be expected. During nucleation $A_{\text {int }}$ of $\sigma\left(\mathrm{CH}_{2}\right)$ adjacent to the $\mathrm{C}=\mathrm{O}$ group appears to be constant (fit gives a small negative rate of $-9.83^{-6}$, but a positive rate is to be expected), whereas $A_{\text {int }}$ of $\sigma\left(\mathrm{CH}_{2}\right)$ for non-trans moieties decreases slightly (rate $-2.06^{-5}$ ) However, a clear distinction between constant rate and the course of the data points cannot be drawn for these vibrations (Fig. 6, Fig. S3 in the Supporting Information).

Garcia and coworkers [32] confirmed that hydrogen bonds in PA66 crystals do not disintegrate below the onset of melting. Consequently, they introduced an "amorphous bound" fraction in addition to an "amorphous free" and "crystalline bound" fraction for their considerations. In accordance with this, Murthy [34] published that a considerable fraction of $\mathrm{H}$ bonds retains even in the melt. As a result, the amorphous 


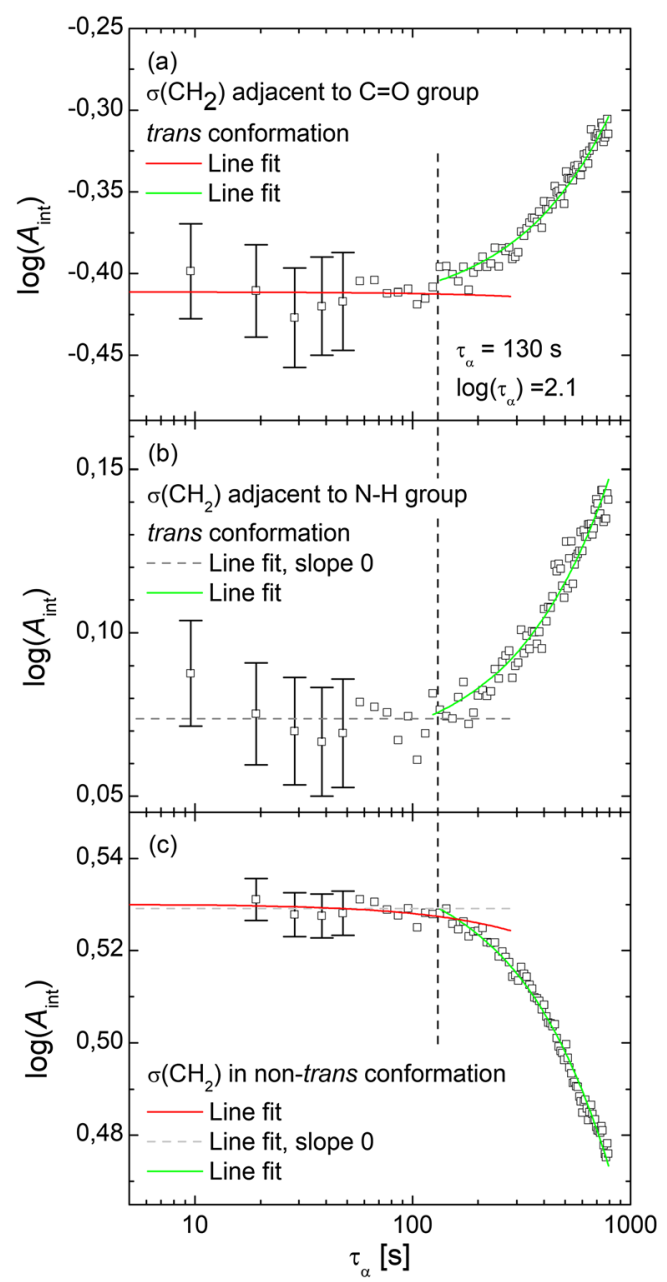

Fig. 6 a Integrated absorbance of the $\mathrm{CH}_{2}$ scissoring vibration $\sigma\left(\mathrm{CH}_{2}\right)$ specific for methylene groups in trans conformation adjacent to the $\mathrm{C}=\mathrm{O}$ side and $\mathbf{b}$ the $\mathrm{N}-\mathrm{H}$ side of the amide group as well as $\mathbf{c}$ the integrated absorbance of $\mathrm{CH}_{2}$ scissoring specific for methylene groups in non-trans conformation. Line fits indicate the constant course and increase of the trans moieties prior and during crystal growth, respectively. All fits are based on data either prior or after the onset of crystal growth (dashed vertical line, compare Fig. 5a)

phase (meant as non-crystalline phase) is not a "shapeless entity"; it can be oriented and the chain packing in this phase can be reformed [34]. This was also demonstrated by Vasanthan and coworker for PA66 fibers up to an order parameter of amorphous chain segments of $\left\langle P_{2}(\cos \theta)\right\rangle=0.6$ [35]. According to Murthy, several attempts have been made in order to designate the amorphous phase, for example, either in terms of isotropic and anisotropic components or mobile and rigid components [41]. Concerning the process of crystallization, Ramesh and coworkers [8] studied the isothermal crystallization of PA66 by means of in situ X-ray diffraction. They successfully demonstrated that crystallization from the melt takes place directly into the (pseudo-hexagonal) Brill structure which is then transformed into the triclinic structure when cooled to room temperature. Furthermore, a substantial temperature interval roughly about $40 \mathrm{~K}$ below the crystallization temperature is found in which the Brill structure exists as a stable modification. Moreover, Vasanthan and coworkers [9] published that $\mathrm{H}$-bonding is not altered in the course of Brill transition and in accord to the references above that $\mathrm{H}$-bonds will remain until the polymer crystals are melted. Thus, there is no doubt, that already in the PA66 melt as well as in the quenched amorphous phase intermolecular H-bonds are well established.

Luo and coworkers [38] employed moving window twodimensional correlation spectroscopy in order to study the step-wise transformation of PA66 during the Brill transition. They demonstrated that the unbound N-H groups change in temporal order before the less-ordered but bound N-H groups and crystalline N-H groups. Thus, the energy benefit is for the crystalline N-H groups as most, which is why they are altered at last. Furthermore, the methylene groups between the amide moieties follow the change of the N-H parts. When comparing this study carried out with increasing temperature and the current work examined the temporal evolution under

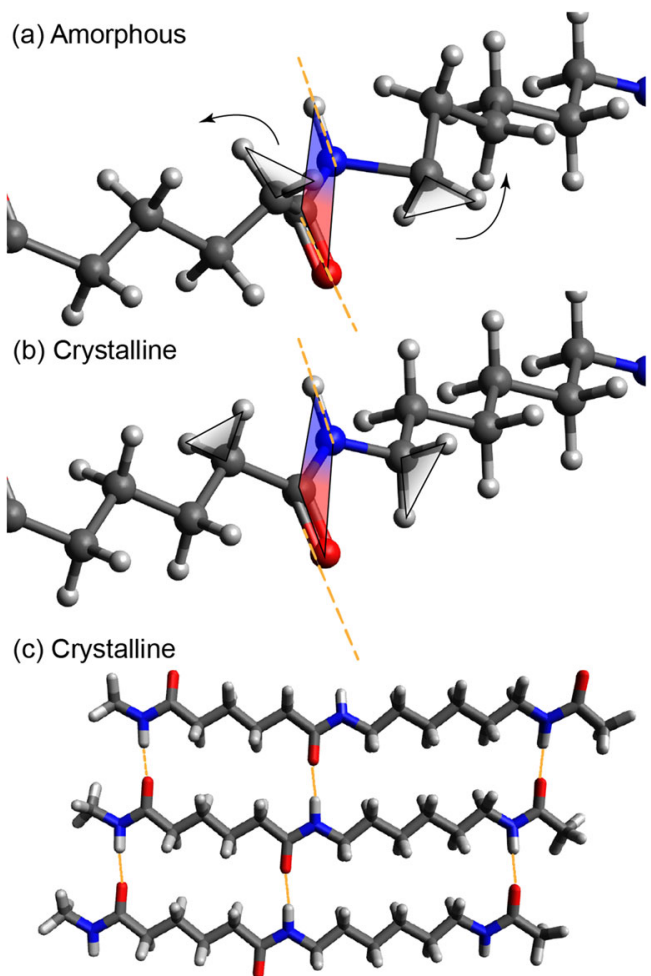

Fig. 7 a It is believed that the N-H parts establish hydrogen bonds (yellow dashed lines) with the $\mathrm{C}=\mathrm{O}$ parts of other amide groups (blue-red quadrilateral) during the course of crystallization. This becomes visible in a slight shift of the N-H single bond vibration frequency (Fig. 3a), but is disguised for the more stable $\mathrm{C}=\mathrm{O}$ double bond (Fig. 3c), because of the strength of this bond compared to the H-bond. b This long range interaction between amide parts forces the methylene groups (gray triangles) between the amide parts to adopt a trans conformation necessary for crystallization and $\mathbf{c}$ a periodic structure. Because the more flexible part as methylene groups are stronger influence through steric constraints, their alteration can be followed in the course of crystallization and to a small extent prior to the onset of crystal growth 
isothermal nucleation and crystal growth, it becomes evident that the results are in reasonable concordance. Intermolecular $\mathrm{H}$-bonds provide the greatest energy benefit and hence are the driving force even in the quenched amorphous state prior to the dominant crystal growth sets in (Figs. 5c and 7a). As a consequence, the methylene groups between the amide parts are forced into a trans conformation in order to enable crystallization (Figs. 6c and 7b), which sets in, when crystal growth becomes the dominating process. During nucleation, the $\mathrm{C}=\mathrm{O}$ groups are not responding (Fig. 5 e), because the $\mathrm{H}$ bonds increase in strength as evident from $v(\mathrm{~N}-\mathrm{H})$, but do not change in conformation. After crystal growth starts, $v(\mathrm{C}=\mathrm{O})$ begins to alter as well. In the case of bending vibrations as $\delta(\mathrm{N}-\mathrm{H})$ (Fig. $5 \mathrm{~g}$ ), transition between crystal nucleation and growth sets in earlier ( $\tau_{\alpha} \approx 60 s$ vs. $130 s$ ) as compared to the H-bond-related stretching vibration $v(\mathrm{~N}-\mathrm{H})$ (Fig. 5c) but also obeys an exponential law at later annealing times.

\section{Conclusion}

On the basis of a combination of fast scanning calorimetry (FSC) and FTIR spectroscopy, we in situ follow the temporal evolution of isothermal crystal nucleation and crystal growth in a thin PA66 film. FSC enables to quench the polymer at $5000 \mathrm{Ks}^{-1}$ into a pure amorphous state. Immediately after quenching, the evolution of the molecular structure is monitored by means of time-dependent FTIR spectroscopy, first during the phase of crystal nucleation and subsequently during crystal growth. It is demonstrated that intermolecular hydrogen bonds between adjacent PA66 chains are established in the melt and persists in the quenched amorphous state where they represent the driving force of structural change in the particular case of PA66. Because polymer mobility is drastically slowed down in the quenched state, the alteration of the different vibrational modes can be followed in detail. The N-H stretching vibration sensitive to the strength of hydrogen bonds is affected already during nucleation prior to the onset of crystal growth, whereas the $\mathrm{C}=\mathrm{O}$ stretching vibration sensitive to conformation is not altered. Both modes exhibit a distinct transition between nucleation and crystal growth regime. Bending vibrations as $\sigma\left(\mathrm{CH}_{2}\right)$ for trans- and non-trans conformers, instead, appear to be constant during nucleation and are forced to alter during crystal growth.

Funding information Open Access funding provided by Projekt DEAL. The authors received support from the following: AMA, German Research Foundation (Deutsche Forschungsgemeinschaft, DFG) project AN 1523/1-1 and German research foundation (Deutsche Forschungsgemeinschaft, DFG) collaborative research center (Sonderforschungsbereich transregio) SFB/TRR 102 project B08; EZ, German research foundation (Deutsche Forschungsgemeinschaft, DFG) priority program SPP 2122 (Project No. ZH 661/3-1); WK, German research foundation (Deutsche Forschungsgemeinschaft, DFG) collaborative research center (Sonderforschungsbereich transregio) SFB/TRR 102 project B08; RA, Ministry of Education and Science of the Russian Federation, grant 14.Y26.31.0019; CS, Ministry of Education and Science of the Russian Federation, grant 14.Y26.31.0019; FK, German research foundation (Deutsche Forschungsgemeinschaft, DFG) collaborative research center (Sonderforschungsbereich transregio) SFB/TRR 102 project B08.

\section{Compliance with ethical standards}

Conflict of interest The authors declare that they have no conflict of interest.

Open Access This article is licensed under a Creative Commons Attribution 4.0 International License, which permits use, sharing, adaptation, distribution and reproduction in any medium or format, as long as you give appropriate credit to the original author(s) and the source, provide a link to the Creative Commons licence, and indicate if changes were made. The images or other third party material in this article are included in the article's Creative Commons licence, unless indicated otherwise in a credit line to the material. If material is not included in the article's Creative Commons licence and your intended use is not permitted by statutory regulation or exceeds the permitted use, you will need to obtain permission directly from the copyright holder. To view a copy of this licence, visit http://creativecommons.org/licenses/by/4.0/.

\section{References}

1. Rhoades AM, Williams JL, Androsch R (2015) Crystallization kinetics of polyamide 66 at processing-relevant cooling conditions and high supercooling. Thermochim Acta 603:103-109

2. Wurm A, Zhuravlev E, Eckstein K, Jehnichen D, Pospiech D, Androsch R, Wunderlich B, Schick C (2012) Crystallization and homogeneous nucleation kinetics of poly ( $\varepsilon$-caprolactone) (PCL) with different molar masses. Macromolecules 45:3816-3828

3. Gibbs JW (1874-1878) On the equilibrium of heterogeneous substances. Trans Conn Acad Arts Sci 3:108-248 and 343-524

4. Fokin VM, Cabral AA, Reis RMCV, Nascimento MLF, Zanotto ED (2010) Critical assessment of DTA-DSC methods for the study of nucleation kinetics in glasses. J Non-Cryst Solids 356:358-367

5. Vyazovkin S, Dranca I (2007) Effect of physical aging on nucleation of amorphous indomethacin. J Phys Chem B 111:7283-7287

6. Sánchez MA, Mathot VBF, Poel GV, Ribelles JLG (2007) Effect of the cooling rate on the nucleation kinetics of poly(L-lactic acid) and its influence on morphology. Macromolecules 40:7989-7997

7. Androsch R, Schick C (2015) Crystal nucleation of polymers at high supercooling of the melt. In: Auriemma F, Alfonso G, de Rosa C (eds) Polymer crystallization I. Advances in polymer science, vol 276. Springer, Cham, pp 257-288

8. Schick C, Androsch R, Schmelzer JWP (2017) Homogeneous crystal nucleation in polymers. J Phys Condens Matter 29:453002

9. Adamovsky SA, Minakov AA, Schick C (2003) Scanning microcalorimetry at high cooling rate. Thermochim Acta 403:55-63

10. Minakov AA, Mordvintsev DA, Schick C (2005) Isothermal reorganization of poly(ethylene terephthalate) revealed by fast calorimetry $\left(1000 \mathrm{Ks}^{-1} ; 5 \mathrm{~ms}\right)$. Faraday Discuss 128:261-270

11. Minakov AA, Schick C (2007) Ultrafast thermal processing and nanocalorimetry at heating and cooling rates up to $1 \mathrm{MK} / \mathrm{s}$. Rev Sci Instrum 78:073902

12. van Drongelen M, Meijer-Vissers T, Cavallo D, Portale G, Poel GV, Androsch R (2013) Microfocus wide-angle X-ray scattering 
of polymers crystallized in a fast scanning chip calorimeter. Thermochim Acta 563:33-37

13. Kolesov IS, Androsch R, Radusch H-J (2004) Non-isothermal crystallization of polyethylenes as function of cooling rate and concentration of short chain branching. J Therm Anal Calorim 78:885-895

14. Zhuravlev E, Schmelzer JWP, Wunderlich B, Schick C (2011) Kinetics of nucleation and crystallization in poly( $\varepsilon$-caprolactone) (PCL). Polymer 52:1983-1997

15. Griffiths PR, de Haseth JA (2007) Fourier transform infrared spectrometry2nd edn. Wiley, Hoboken

16. Siesler HW (2011) Vibrational spectroscopy of polymers. Int $\mathbf{J}$ Polym Anal Charact 16:519-541

17. Tress M, Anton AM, Vielhauer M, Lutz P, Mülhaupt R, Kremer F (2020) Confined glassy dynamics in a star-shaped polymer induced by crystallization: case study of polyhedral oligomeric polysilesquioxane - isotactic polystyrene (POSS-iPS). In: Ezquerra T (ed) Dielectrics and crystallization. Advances in dielectrics. Springer, Cham Chapter 11, in press

18. Zhuravlev E, Schick C (2010) Fast scanning power compensated differential scanning nano-calorimeter: 1 . The device. Thermochim Acta 505:1-13

19. Cebe P, Hu X, Kaplan DL, Zhuravlev E, Wurm A, Arbeiter D, Schick C (2013) Beating the heat - fast scanning melts silk beta sheet crystal. Sci Rep 3:1130

20. Androsch R, Schick C, Rhoades AM (2015) Application of Tammann's two-stage crystal nuclei development method for analysis of the thermal stability of homogeneous crystal nuclei of poly(ethylene terephthalate). Macromolecules 48:8082-8089

21. Zhuravlev E, Schmelzer JWP, Abyzov AS, Fokin VM, Androsch R, Schick C (2015) Experimental test of Tammann's nuclei development approach in crystallization of macromolecules. Cryst Growth Des 15:786-798

22. Androsch R, Schick C (2016) Interplay between the relaxation of the glass of random L/D-lactide copolymers and homogeneous crystal nucleation: evidence for segregation of chain defects. $\mathrm{J}$ Phys Chem B 120:4522-4528

23. Androsch R, Zhang R, Schick C (2019) Melt-recrystallization of poly (L-lactic acid) initially containing $\alpha^{\prime}$-crystals. Polymer 176: 227-235

24. Stancik AL, Brauns EB (2008) A simple asymmetric lineshape for fitting infrared absorption spectra. Vib Spectrosc 47:66-69

25. Gao S, Simon SL (2015) Measurement of the limiting fictive temperature over five decades of cooling and heating rates. Thermochim Acta 603:123-127

26. Tropin TV, Schulz G, Schmelzer JWP, Schick C (2015) Heat capacity measurements and modeling of polystyrene glass transition in a wide range of cooling rates. J Non-Cryst Solids 409:63-75

27. Brucato V, Crippa G, Piccarolo S, Titomanlio G (1991) Crystallization of polymer melts under fast cooling. I: nucleated polyamide 6. Polym Eng Sci 31:1411-1416

28. Minakov AA, Mordvintsev DA, Tol R, Schick C (2006) Melting and reorganization of the crystalline fraction and relaxation of the rigid amorphous fraction of isotactic polystyrene on fast heating $(30,000 \mathrm{~K} / \mathrm{min})$. Thermochim Acta 442:25-30

29. Minakov AA, Mordvintsev DA, Schick C (2004) Melting and reorganization of poly(ethylene terephthalate) on fast heating (1000 $\mathrm{K} / \mathrm{s}$ ). Polymer 45:3755-3763

30. Cooper SJ, Coogan M, Everall N, Priestnall I (2001) A polarized $\mu-$ FTIR study on a model system for nylon 6: implications for the nylon Brill structure. Polymer 42:10119-10132

31. Ma Y, Zhou T, Su G, Li Y, Zhang A (2016) Understanding the crystallization behavior of polyamide 6/polyamide 66 alloys from the perspective of hydrogen bonds: projection moving-window $2 \mathrm{D}$ correlation FTIR spectroscopy and the enthalpy. RSC Adv 6: 87405-87415
32. Garcia D, Starkweather Jr HW (1985) Hydrogen bonding in nylon 66 and model compounds. J Polym Sci 23:537-555

33. Skrovanek DJ, Painter PC, Coleman MM (1986) Hydrogen bonding in polymers. 2. Infrared temperature studies of nylon 11. Macromolecules 19:699-705

34. Murthy NS (2006) Hydrogen bonding, mobility, and structural transitions in aliphatic polyamides. J Polym Sci Part B Polym Phys 44: 1763-1783

35. Vasanthan N, Ruetsch SB, Salem DR (2002) Structure development of polyamide-66 fibers during drawing and their microstructure characterization. J Polym Sci Part B Polym Phys 40:19401948

36. Ramesh C, Keller A, Eltink SJEA (1994) Studies on the crystallization and melting of nylon-6,6: 1. The dependence of the Brill transition on the crystallization. Polymer 35:2483-2487

37. Vasanthan N, Murthy NS, Bray RG (1998) Investigation of brill transition in Nylon 6 and Nylon 6,6 by infrared spectroscopy. Macromolecules 31:8433-8435

38. Luo J, Zhou T, Fu X, Liang H, Zhang A (2011) Mechanism in Brill transition of polyamide 66 studied by two-dimensional correlation infrared spectroscopy. Eur Polym J 47:230-237

39. Zhuravlev E, Wurm A, Pötschke P, Androsch R, Schmelzer JWP, Schick (2014) Kinetics of nucleation and crystallization of poly(ecaprolactone) - multiwalled carbonnanotube composites. Eur Polym J 52:1-11

40. Androsch R, Schick C, Schmelzer JWP (2014) Sequence of enthalpy relaxation, homogeneous crystal nucleation and crystal growth in glassy polyamide 6. Eur Polym J 53:100-108

41. Bruna P, Crespo D, González-Cincab R (2006) On the validity of Avrami formalism in primary crystallization. J Appl Phys 100: 054907

Publisher's note Springer Nature remains neutral with regard to jurisdictional claims in published maps and institutional affiliations.

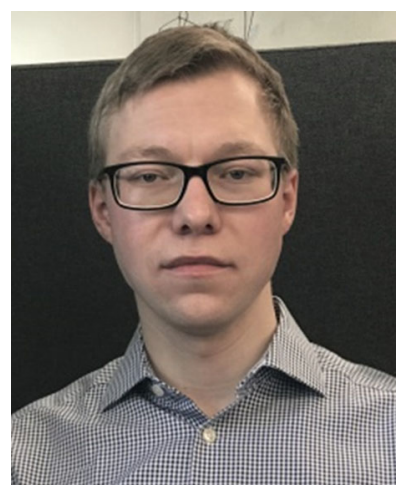

A. Markus Anton received his diploma in 2011 from the Technische Universität Dresden (TUD, Germany) under supervision of Prof. Schwille and Dr. Petrov in the Biophysics Group and his Dr. rer. nat. in 2016 from Leipzig University (Germany) under supervision of Prof. Kremer in the Molecular Physics Group. After continuing working as a postdoc at Leipzig University, he joined the Fast Spectroscopy Group headed by Dr. Clark at the University of Sheffield (UK) as postdoctoral fellow in 2019. He is interested in polymer and molecular physics, especially in the spatial orientation and interaction of energy-related polymers and molecules such as conjugated polymers or (polymerized) ionic liquids. The central experimental methods used in his research are spectroscopy with light, mainly in combination with external stimuli such as temperature-, stress-, or pressure-dependent measurements, orientation-dependent measurements, as well as measurements by means of frequency- and temperature-dependent Broadband Dielectric Spectroscopy (BDS). 


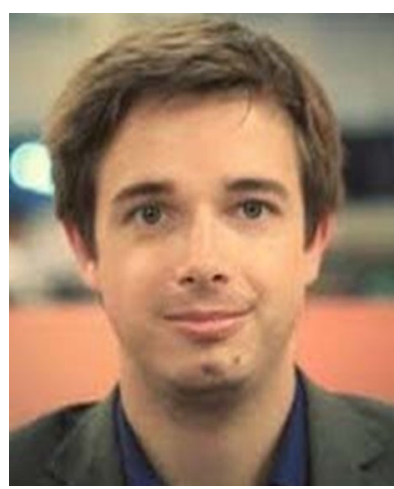

E. Zhuravlev is a postdoc in University of Rostock, heading the laboratory of Fast Scanning Calorimetry in competence center ${ }^{\circ}$ CALOR. He graduated in applied physics and mathematics in 2006 in MIPT (Russia) and received his $\mathrm{PhD}$ in 2010 at the University of Rostock in experimental physics. From 2006 till 2019, he was doing research in the polymer physics AG of Prof. Christoph Schick. During this period, he developed a Fast Scanning Calorimetry and applied it to study of polymers, metals, and nanocomposite solidification and melting. In the period from 2016 till 2018, he worked as invited researcher in Nanjing University (China), Group of Polymer Physics and Engineering under supervision, Prof. Dongshan Zhou. Currently, he is working on the melting and solidification of polymers and metals under laser heating conditions for Additive Manufacturing.

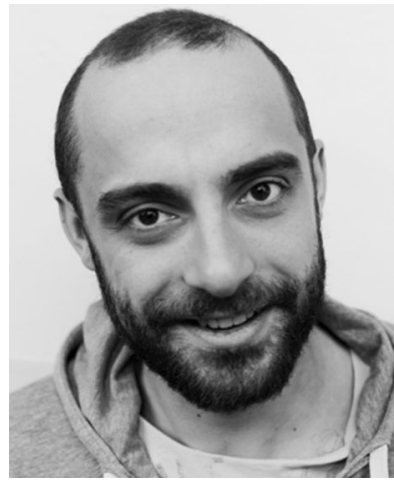

W. Kossack was a postdoctoral fellow at Leipzig University in the research group of Molecular Physics under Prof. Kremer. He graduated in 2015 on structure and properties of polymers using advanced IR spectroscopic techniques. Working on semicrystalline and glassy polymers, he (co-) authored several research papers and book chapters utilizing IR spectroscopy among a multitude of other approaches. In 2019 , he took over a position in industry.

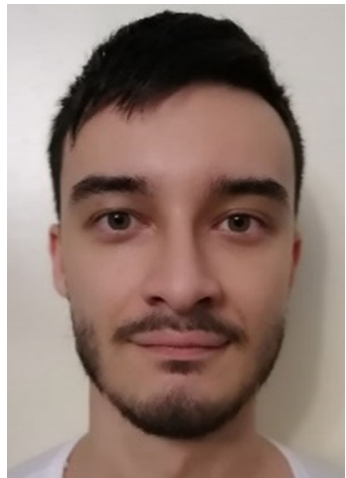

Ruslan Andrianov is currently a first-year graduate student in the Department of Physical Chemistry of Kazan Federal University, Russia. He wrote diploma thesis about investigation of kinetics of nucleation and crystallization of polymers with using fast scanning calorimetry and successfully defended it. His areas of interest are calorimetry and its application in the study of polymer reorganization processes.

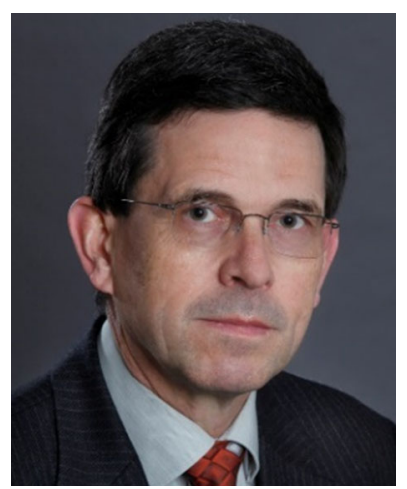

Christoph Schick was Professor and Head of the Polymer Physics Group at the Institute of Physics of the University of Rostock, Germany, 1992-2019. Since 2017, he holds a part-time professorship at Kazan Federal University, Russia. He studied Polymer Physics at the Technische Hochschule LeunaMerseburg, Germany, and received his $\mathrm{PhD}$ degree in 1980 for research in the field of glass transition. As a postdoctoral research associate, he became interested in the interplay between glass transition and crystallization in polymers. In this field, he completed his Habilitation in 1988. Since then, his research focuses on advanced calorimetry like fast scanning methods, applied to polymers, metals, biological molecules, and other substances. $\mathrm{He}$ is interested in crystallization, nucleation, and glass transition in these materials.

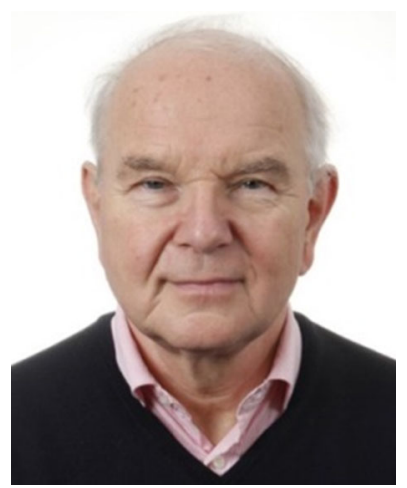

Friedrich Kremer is professor emeritus at Leipzig University, where he held the chair for Molecular Physics from 1993 to 2015. He graduated in theoretical physics (LMU Munich) and received his $\mathrm{PhD}$ at the University of Freiburg in experimental biology. In 1978-1985, he joined the Max-Planck-Institute (MPI) for solid state physics (Stuttgart), and in 1985-1993, the MPI for polymer research (Mainz). From 1999 to 2003, he was chairman of the "Chemical and Polymer Physics Division" of the German Physical Society (DPG). His current research interests are Broadband Dielectric Spectroscopy and refined methods of Fourier-transform infrared spectroscopy. He authored or edited several books and published more than 390 papers in peerreviewed journals and is chief advisory editor of "Colloid and Polymer Science". In 2005, he received the Karl Heinz Beckurts Award for his achievements in promoting Broadband Dielectric Spectroscopy and its applications and in 2011 the Wolfgang Ostwald Prize of the German Colloid Society.

Dear Matthias, this work also includes a message beyond nucleation and crystallization: Be always aware of the reach of your interactions! 